\title{
Tagungsbericht
}

\section{Technikgeschichtliche Jahrestagung des VDI 2013}

\author{
"Technik im Kalten Krieg II (1970-1990)" \\ am 14. und 15. Februar 2013 in Bochum
}

VON LARS BLUMA

\begin{abstract}
Wasserkocher, Mikroelektronik, Spionage, Flexible Response, Technikverdrossenheit und Umweltproteste - mit diesen Schlagworten lässt sich das weite Themenspektrum der diesjährigen technikgeschichtlichen Jahrestagung des VDI in Bochum umreißen. Anknüpfend an die letztjährige Tagung des VDI zur Technikentwicklung in den ersten beiden Jahrzehnten des Kalten Krieges, rückten nun die 1970er und 1980er Jahre in den Fokus der Vorträge. Die inhaltliche Heterogenität der Fallbeispiele mit Beiträgen zur Rüstungsforschung, zur Produktionstechnik und zum Konsum in diesen beiden Jahrzehnten machte von Anfang an neugierig, wie und ob sich die Einzelteile zu einem Ganzen fügen würden - doch dazu später.

Im Eröffnungsvortrag gab Karin Zachmann (München) einen profunden Überblick über die enge Verflechtung von Technik, Politik und Kultur während der gesamten Phase des Kalten Krieges. Anschließend an aktuelle Debatten in der internationalen Zeitgeschichtsforschung mahnte sie an, technische Entwicklungen in dieser Zeit nicht ausschließlich unter dem Gesichtspunkt der historischen Systemkonfrontation und der politischen Bipolarität der Welt zu analysieren. Sowohl im Hinblick auf die globalen geographisch-räumlichen Strukturen (man beachte nur die Rolle der sogenannten Entwicklungsländer) als auch auf die Entstehung neuer sozialer Bewegungen nach dem Zweiten Weltkrieg forderte sie eine differenziertere Betrachtung ein. Als zentrales Thema einer Technikgeschichte des Kalten Krieges benannte sie die Hybridisierung von Wissen, Technik und Politik. Das Spezifische des Kalten Krieges sah Zachmann in der intensiven Verschränkung bzw. der Ko-Konstruktion von technisch-materiellen Strukturen und dem politischen Handeln. Sie spitzte dies sogar mit der These zu, dass für den untersuchten Zeitraum die materiellen Strukturen als Prämissen der Politik anzusehen seien. Insbesondere die Atomwaffen schufen eine gleichsam nicht hintergehbare technisch-materielle Struktur für das politische Handeln der Akteure.
\end{abstract}


Nach diesem Überblicksvortrag stellte Sibylle Marti (Zürich) mit einem Vortrag über die nuklearen Kriegsszenarien in der Schweiz die erste Fallstudie der VDI-Tagung vor. Mit kybernetisch orientierten Szenariotechniken versuchte das schweizerische Militär seit den 1970er Jahren die Folgen eines Nuklearkrieges durchzuspielen, um dadurch Rückschlüsse für eine angemessene Organisation der Gesamtverteidigung zu gewinnen. Neben dieser ganz praktischen Funktion für die militärische Landesverteidigung wurde mit den Kriegsszenarien ein Instrument entwickelt, mit dem eine Nuklearkatastrophe beherrschbar schien. Voraussetzung hierfür war eine permanente gesellschaftliche Selbstbeobachtung, die den planerischen Soll- mit dem tatsächlichen Ist-Zustand der Verteidigungsmaßnahmen, die letztendlich alle ökonomischen Ressourcen der Schweiz ins Kalkül zog, permanent abglich. Diese Rationalisierungsstrategie scheiterte jedoch letztendlich an den Ergebnissen der Szenarien selbst, die eine nahezu vollständige Vernichtung der Schweiz im Falle eines Atomkriegs prognostizierten. Von einer Beherrschung der Risiken eines Nuklearkriegs konnte also keine Rede mehr sein.

Von diesem ernüchternden Ergebnis der schweizerischen Szenariotechnik recht unbeeindruckt, entwickelte die NATO im Laufe des Kalten Krieges unterschiedliche Strategien der Kriegsführung. Seit dem Ende der 1960er Jahre galt die sogenannte „Flexible Response“ als verbindliche Strategie, die im Gegensatz zur Doktrin der ,,massiven Vergeltung“ differenzierte und abgestufte Maßnahmen im Falle eines sowjetischen Angriffs vorsah. Wie Dieter Kollmer (Potsdam) in seinem Vortrag schlüssig zeigen konnte, führte die „Flexible Response" in Westdeutschland zu einer militärtechnischen Sonderentwicklung. Nicht nur, dass sich die Waffenentwicklung und Rüstungsbeschaffung der BRD auf konventionelle Waffensysteme konzentrierte, um die Bundeswehr in die Lage zu versetzen, einen massiven konventionellen Angriff des Warschauer Paktes an der innerdeutschen Grenze zu begegnen. Vielmehr verhinderte diese Spezialisierung auf konventionelle Kriegsführung die Entstehung eines militärisch-industriellen Komplexes, wie er in den USA zu beobachten war. Anders als in den USA zeichnete sich die Beschaffungspolitik der Bundeswehr durch eine geringe Verflechtung von Militär und Waffenherstellern aus, was sich auch, so Kollmer, im öffentlich kontrollierten Vergabeverfahren der Bundeswehr widerspiegelte.

Auch wenn sich in der Bundesrepublik kein militärisch-industrieller Komplex wie in den USA etablieren konnte, gerieten die erheblichen staatlichen Rüstungsausgaben und die Rüstungsbranche seit den 1970er Jahren verstärkt in die Kritik der westdeutschen Öffentlichkeit. Mit der Formierung der Friedensbewegung wurden gesellschaftliche Alternativen zur Rüstungsproduktion entwickelt. Mithin beinhalteten diese neuen gesellschaftlichen Ordnungsvorstellungen auch Konzepte einer alternativen Produktion als zentrales Element, die im Slogan „Statt Waffen nützliche Dinge“ mündeten. Anne Sudrows (Potsdam) Beitrag zeigte am Beispiel des englischen 
Luftfahrtkonzerns Lucas Aerospace sehr eindrucksvoll, wie das Konzept der „sozial nützlichen Produkte“ als basisdemokratische Bewegung von gewerkschaftlich organisierten Ingenieuren, Technikern und Arbeitern innerhalb einzelner Betriebe entwickelt wurde und sich durch öffentlichkeitswirksame Aktionen in ganz Westeuropa verbreitete. Die Forderung, die Produktion von Rüstungsgütern durch die Produktion von ,nützlichen Dingen“ zu ersetzen, führte zu betriebspraktischen Experimenten und zahlreichen konkreten Konversionsvorschlägen, die im Kern der Umwelttechnik in den 1980er Jahren den Weg bahnen sollten. Damit waren die englischen Industriebelegschaften, die das Konzept der ,alternativen Produktion“ vertraten, Wegbereiter einer neuen ökonomischen Ordnung, die die auf Schwerindustrie basierende alte ökonomische Ordnung ablösen sollte, so die These Anne Sudrows.

Oliver Schmidt (Mannheim) eröffnete mit seinem Vortrag über Haushaltstechnik im Systemwettlauf den zweiten Tag der Tagung. Die Versorgung der Bevölkerung mit Konsumgütern war seit den 1970er Jahren eine Notwendigkeit in Ost und West, um die Legitimation der eigenen Staats- und Wirtschaftsform zu sichern. Konsum wurde nun zu einem Gradmesser der Über- oder Unterlegenheit der politischen Rivalen und damit neben der Militärtechnik und der Raumfahrt zu einem weiteren „Schlachtfeld“ des Kalten Krieges. Schmidt wählte einen objektorientierten Ansatz, der Design und Funktionalität von west- und ostdeutschen Konsumgütern, wie Wasserkocher, Waschmaschinen und Tischventilatoren, miteinander vergleicht. Tatsächlich konnte der Referent zunächst keine technischen und qualitativen Unterschiede zwischen west- und ostdeutschen Konsumgütern ausmachen, vielmehr verkauften westdeutsche Unternehmen häufig Ostprodukte, die einfach ein neues Label erhielten. Diese gedeihliche Zusammenarbeit lässt allerdings an der Grundthese des Vortrags, dass die Konsumgüterindustrie zu einem Schlachtfeld des Kalten Krieges wurde, erhebliche Zweifel aufkommen.

Mit dem anschließenden Vortrag von Frank Dittmann (München) verließ die Tagung den Bereich der Konsumgüter und wendete sich einer weiteren industriepolitisch und militärstrategisch wichtigen Technologiebranche zu, der Mikroelektronik. Im Zentrum des Vortrags stand die Frage, ob die DDR die komplexe Fertigung integrierter Schaltungen beherrschte, oder ob entsprechende staatliche Erfolgsmeldungen als Propaganda anzusehen sind. Mit dem westlichen Embargo Anfang der 1980er Jahre befand sich die DDR in einer Zwangssituation, da ihre Wirtschaft zumindest im Bereich der Mikroelektronik von der internationalen Arbeitsteilung abgeschottet war. Mit Wirtschaftsspionage, geheimen Technologietransfers und Schmuggel versuchte die DDR dieses Problem zu kompensieren. Allerdings, so das Resümee von Dittmann, konnten die ostdeutschen Ingenieure sehr wohl die westliche Mikroelektronik nachbauen, die innovative Weiterentwicklung derselben misslang aber, sodass 1988 in der politischen Führung der DDR selbst Zweifel am Erfolg einer eigenen Mikroelektronikindustrie aufkamen. 
Dem Thema der Industriespionage widmete sich auch Miroslaw Sikoras (Katowice) Beitrag über die Rolle des Geheimdienstes für die Forschung und Entwicklung von Technik in Polen. Ähnlich wie Dittmann sah er das Embargo der Westmächte, das den Zugang der polnischen Industrie zu moderner Technologie erheblich einschränkte, als entscheidende Zäsur für die Technologieentwicklung an. Der polnische Geheimdienst mutierte in den 1980er Jahren immer mehr von einem klassischen Aufklärungsdienst zu einem Beschaffungsdienst, der, eng integriert in das Industrieministerium, als gewinnorientiertes, kapitalistisches Unternehmen agierte und für die polnische Industrie Technologie und Informationen beschaffte.

In der Bundesrepublik beschäftigten andere Themen die Öffentlichkeit. Wie Andie Rothenhäusler (Karlsruhe) darstellte, kam in den 1980er Jahren eine heftig geführte Debatte um die vermeintliche Technikfeindlichkeit der Bevölkerung auf. Die von Umfragen befeuerte Auseinandersetzung, die auch die zeitgenössische Soziologie ergriff, kreiste um die Frage, ob die konstatierte Technikfeindlichkeit eine Bedrohung für den Wohlstand des Landes darstellte. Technikfeindlichkeit wurde nun ein medial-politischer Kampfbegriff, der eben nicht der Beschreibung der Wirklichkeit diente, sondern politisch instrumentalisiert wurde. Technikkritik in allen Abstufungen und Schattierungen konnte nun desavouiert werden mit der Unterstellung, dass eine skeptische Einstellung zur Technik zurück in die technologische Steinzeit führen würde. Während diese Debatte relativ schnell wieder abebbte, erfahren ähnliche Argumentationsmuster aktuell wieder eine Renaissance.

In diesem wie auch im folgenden Beitrag blieb allerdings der Bezug zum Kalten Krieg recht vage. Technikverdrossenheit und auch die einsetzenden Umweltproteste, die Thilo Jungkind (Rottweil am Neckar) im anschließenden Vortrag am Beispiel der Proteste gegen die Dünnsäureverklappung thematisierte, scheinen eher einem allgemeinen soziokulturellen Wandel in den 1980er Jahren geschuldet zu sein, der nur noch sehr gering von der Blockkonfrontation beeinflusst war. Jungkinds unternehmenshistorischer Ansatz zeigte jedoch, wie die Wahrnehmungsmächtigkeit der neu entstehenden Risikosemantik die Risikomentalität der Akteure und das daraus abgeleitete unternehmerische Störfallverhalten beeinflussten. Die öffentlichen Proteste gegen die Dünnsäureverklappung schufen für das Unternehmen Bayer einen Anreiz zur Findung neuer Technologien, und mit der Radikalisierung der Umweltproteste nach der Seveso-Katastrophe 1976 entwickelte Bayer Recyclingverfahren, die die Verklappung der Dünnsäure in der Nordsee überflüssig machten. Damit konnte der Referent den Einfluss der institutionellen Neuformierung der BRD auf die technologische Entwicklung seit den 1970er Jahren zumindest für den untersuchten Einzelfall empirisch nachweisen.

So interessant die Einzelbeiträge und die jeweiligen Fallstudien, die auf der VDI-Tagung vorgestellt wurden, auch waren, eine Epochenbeschreibung der 1970er und 1980er Jahre in einer dezidiert technikhistorischen Perspektive 
lieferten diese allerdings nicht. Andeutungsweise ließe sich der Aufstieg der EDV- und Konsumtechnik als prägende Elemente aus den Fallstudien herausfiltern. Doch was sind die leitenden Fragestellungen und Thesen der Technikgeschichte zur zweiten Phase des Kalten Krieges? Wo sind technikhistorische Kontinuitäten und Brüche auszumachen, die diesen Zeitraum prägten? Lässt sich unter technikhistorischen Gesichtspunkten überhaupt eine einheitliche Epoche zwischen 1970 und 1989 ausmachen? Karin Zachmann hat mit ihrer Ausführung zur Hybridisierung von Technik, Politik und Gesellschaft zwar ein interessantes methodisch-theoretisches Angebot gemacht, welches explizit eine enge Verknüpfung von Technik- und Zeitgeschichte einforderte, allerdings folgten die anderen Referenten der Tagung diesem Weg nur bedingt. Zumindest Anne Sudrow hat sich in ihrem Vortrag auf aktuelle Positionen der Zeitgeschichte bezogen, insbesondere auf die Problematik der ökonomischen Transformation nach der Boomphase der 1950er und 1960er Jahre.

Alles in allem gewann man allerdings den Eindruck, dass sich die Vortragenden nicht auf die momentan in der Zeitgeschichte intensiv geführte Debatte über die Charakterisierung der Jahre „nach dem Boom“ eingelassen haben. Vor allen Dingen wäre zu hinterfragen, ob die Blockkonfrontation in den 1970er und 1980er Jahre überhaupt noch eine strukturprägende Kraft auf die technische Entwicklung hatte, oder ob nicht andere historische Prozesse, die die Geschichtswissenschaft mit Begriffen wie Strukturwandel, dritte industrielle Revolution, Postfordismus, Postmoderne o.ä. auf den Punkt zu bringen versucht, nicht weitaus einflussreicher waren. Und das gilt es sowohl für die kapitalistisch als auch planwirtschaftlich organisierten Ökonomien gleichermaßen zu diskutieren und zu klären.

Anschrift des Verfassers: Dr. Lars Bluma, Deutsches Bergbau-Museum Bochum, Am Bergbaumuseum 28, 44791 Bochum, Email: lars.bluma@ bergbaumuseum.de 


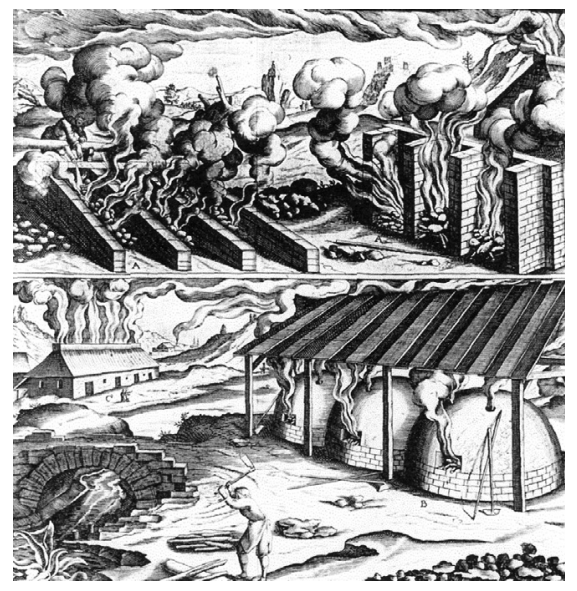

\section{Unschlagbild}

Das Umschlagbild zeigt den Vorgang des Röstens zur Entschwefelung sulfidischer Erze vor dem Probieren und Schmelzen. Stückiges Erz wurde der Haufenröstung (in der Regel im Freien) unterzogen, feinkörnige sulfidische Erze durch Luftzutritt im Röstofen (in gemauerten Stadeln oder in überdachten Röstschuppen) bearbeitet. Der Sulfid verbrannte zu Schwefeldioxid $\left(\mathrm{SO}_{2}\right)$ und entwich in dieser Form gasförmig. Quelle: Georg Engelhardt Löhneiß, Gründlicher und ausführlicher Bericht vom Bergwerk, Zellerfeld 1617.

Hans-Joachim Kraschewski 